\title{
P2Y12 inhibition in macrophages reduces ventricular arrhythmias in rats after myocardial ischemia-reperfusion
}

\author{
Lu Wang ${ }^{1,2, A-F}$, Na Li3 B, B, ,E,F, Fei Wang 2,B,C,E,F, Lianqun Cui ${ }^{1, A, F}$ \\ ${ }^{1}$ Department of Cardiology, Shandong Provincial Hospital, Cheeloo College of Medicine, Shandong University, Jinan, China \\ 2 Department of Internal Medicine, Shandong Corps Hospital of Chinese People's Armed Police Forces, Jinan, China \\ ${ }^{3}$ Outpatient Department, Shandong Corps Hospital of Chinese People's Armed Police Forces, Jinan, China \\ A - research concept and design; B - collection and/or assembly of data; C - data analysis and interpretation; \\ $D$ - writing the article; $E$ - critical revision of the article; $F$ - final approval of the article
}

\section{Address for correspondence}

Lianqun Cui

E-mail: cuilianqunsdslyy@163.com

Funding sources

None declared

Conflict of interest

None declared

Received on October 20, 2020

Reviewed on December 28, 2020

Accepted on February 7,2021

Published online on April 28, 2021

\begin{abstract}
Background. Myocardial ischemia-reperfusion (I/R) injury is still thought to be an unsolved puzzle that may lead to reperfusion arrhythmias and sudden cardiac death. Inflammation plays a key role in myocardial I/R. Studies have indicated that purinoceptor 2 Y12 (P2Y12) antagonists have anti-inflammatory properties that are cardioprotective.
\end{abstract}

Objectives. In this study, we explored whether inhibition of P2Y12 in macrophages could reduce cardiac inflammation and attenuate reperfusion arrhythmias after myocardial I/R.

Materials and methods. Rats were randomly divided into 4 groups: group A (control + vehicle); group B (control + P2Y12 shRNA lentiviral vector); group C (myocardial I/R + vehicle); and group D (myocardial I/R + P2Y12 shRNA lentiviral vector). Infarct size, reperfusion arrhythmias, and P2Y12 and platelet endothelial cell adhesion molecule-1 (CD31) protein expression were measured.

Results. The incidence of reperfusion ventricular tachycardia and fibrillation (VT/VF) was $90 \%$ in the I/R group, while it was reduced to 50\% by P2Y12 shRNA treatment. Ionized calcium binding adapter molecule 1 and P2Y12 immunoreactivity in the myocardial I/R + P2Y12 shRNA group was lower compared to the myocardial I/R group. P2Y12 shRNA treatment increased a-smooth muscle actin (a-SMA) and CD31 protein expression, as evidence by western blot and immunohistochemistry analyses $(0.31 \pm 0.01$ compared to $0.26 \pm 0.008$, group D compared to group $C, p<0.05$ ).

Conclusions. Inhibition of P2Y12 in macrophages improved reperfusion arrhythmias in our rat I/R model, suggesting that blocking P2Y12 could decrease the inflammatory response after cardiac perfusion.

Key words: macrophages, inflammation, P2Y12, myocardial ischemia-reperfusion, reperfusion arrhythmia

Cite as

Wang L, Li N, Wang F, Cui L. P2Y12 inhibition in macrophages reduces ventricular arrhythmias in rats after myocardial ischemia-reperfusion. Adv Clin Exp Med. 2021;30(4):413-420. doi:10.17219/acem/133139

DOI

10.17219/acem/133139

Copyright

Copyright by Author(s)

This is an article distributed under the terms of the

Creative Commons Attribution 3.0 Unported (CC BY 3.0)

(https://creativecommons.org/licenses/by/3.0/) 


\section{Background}

Acute myocardial infarction (AMI) is one of the most common causes of cardiovascular death worldwide. Reperfusion is the primary mechanism to restore blood flow to the heart in the treatment of AMI. However, myocardial ischemia-reperfusion (I/R) can cause a variety of injuries through a number of processes, such as cell and organelle membrane injury, oxidative stress, endothelial injury, vasoconstriction, and pro-inflammatory immune responses. ${ }^{1}$ Accordingly, reperfusion can cause heart tissue damage, electrophysiological dysfunction and heart failure. ${ }^{2,3}$ One example are reperfusion arrhythmias, particularly ventricular tachycardia and fibrillation (VT/VF), which are serious events that complicate reperfusion therapies, like coronary interventions and thrombolytic therapy, in patients with AMI. ${ }^{4}$ Sympathetic over-stimulation and inflammation are 2 major mediators of reperfusion arrhythmias. ${ }^{5}$ Therefore, reducing cardiac inflammation could be an effective means to prevent myocardial I/R injury and the occurrence of reperfusion arrhythmias. Inflammation is a part of the physiological wound-healing response after mechanical injuries. At the onset of an infarction, a strong inflammatory response is initiated, ${ }^{6}$ which involves macrophage activation. ${ }^{7}$ Macrophages play an important role in myocardial repair and remodeling after myocardial infarction. However, the role of macrophages in I/R injury remains unclear. Previous studies have shown that the platelet P2Y12 (P2Y12) receptor plays a central role in platelet function, hemostasis and thrombosis, but there have only been a few studies that have explored the non-platelet effects of P2Y12 in cardiovascular diseases. ${ }^{8,9}$ One study demonstrated that inhibiting the P2Y12 receptor reduced the increase in pro-inflammatory mediators, pointing to the cardioprotective properties of P2Y12 antagonists. ${ }^{9}$ In our study, we investigated the role and mechanism of P2Y12 inhibition in I/R injury.

\section{Materials and methods}

\section{Animals}

Sixty 8-week-old male Sprague Dawley rats weighing 200-270 g were purchased from the Vital River Laboratory (Beijing, China). The protocols was approved by the Ethics Committee of Shandong Corps Hospital of Chinese People's Armed Police Forces, Jinan, China, and rats received humane care conforming to the National Institutes of Health Guide for the Care and Use of Laboratory Animals. Rats had free access to normal rat chow and drinking water.

\section{Experimental design}

A total of 60 rats were randomly divided into 4 groups: group A (control + vehicle); group B (control + P2Y12 shRNA lentiviral vector (Santa Cruz Biotechnology,
Santa Cruz, ISA); group C (myocardial I/R + vehicle); and group D (myocardial I/R + P2Y12 shRNA lentiviral vector). Rats received P2Y12 shRNA lentiviral vector via tail vein injection starting 5 days before myocardial I/R surgery. Rats in the sham control groups were administered vehicle. After the shRNA expression vector enters the cytoplasm, the vector needs to be transported to the cytoplasmic nucleus for transcription. ShRNAs are synthesized in the nucleus, processed and transported to the cytoplasm, and finally incorporated into the RNA-interfering silencing complex. Mature shRNA provides RNA interference through mRNA cleavage and degradation. ${ }^{10}$ The incidence and duration of VT/VF in the reperfusion period were recorded to evaluate reperfusion arrhythmias. After sacrifice, heart tissues were collected for western blot and immunohistochemistry analyses.

\section{Myocardial I/R surgery}

Myocardial I/R surgery was performed 5 days after P2Y12 shRNA lentiviral vector injection. After anesthetizing with $3 \%$ pentobarbital sodium $(30 \mathrm{mg} / \mathrm{kg}$; intraperitoneal), intubating via tracheotomy and ventilating with a small animal ventilator, a 3-cm left thoracotomy was performed through the left $4^{\text {th }}$ intercostal space. The left anterior descending (LAD) coronary branch was ligated with 6/0 silk sutures for 30 min to induce myocardial ischemia. During the procedure, we monitored cardiac function using electrocardiogram (ECG) recordings (BL-420S; TaiMeng, Dongguan, China). Myocardial ischemia was confirmed with ST-segment elevation and tall T-waves. Rats in the sham surgery groups underwent only thoracotomy and the LAD coronary was not ligated. After $30 \mathrm{~min}$ of ligation, the LAD coronary was reperfused for $2 \mathrm{~h}$.

\section{Evaluation of arrhythmias}

Arrhythmias were assessed according to the diagnostic criteria of the Lambeth Conventions. ${ }^{9,11}$ Ventricular arrhythmia parameters, including percent incidence of VT/VF and duration of VT/VF, were quantified during the whole reperfusion period. Ventricular tachycardia refers to the occurrence of 4 or more consecutive ventricular premature beats in a row. Ventricular fibrillation is defined as a signal whose individual QRS wave deflections are no longer distinguishable from each other (meaning morphological instability) and whose frequency can no longer be measured.

\section{Determination of myocardial injury}

Myocardial ischemic size was determined using Evans blue/triphenyltetrazolium chloride (TTC) staining. After reperfusion, $1 \%$ Evans blue staining solution $(0.3 \mathrm{~mL})$ was injected to determine the ischemic area. The tissues were then incubated in a $2 \%$ TTC solution (Sigma Aldrich, St. 
Louis, USA) for $30 \mathrm{~min}$ at $37^{\circ} \mathrm{C}$, and fixed in $4 \%$ paraformaldehyde solution overnight. After storing at $-20^{\circ} \mathrm{C}$ for $20 \mathrm{~min}$, the heart was cut into $1-\mathrm{mm}$ thick slices. The myocardium in the ischemic area and infarction area was stained blue and light yellow, respectively.

\section{Immunofluorescence}

Hearts were sectioned into approx. 7- $\mu \mathrm{m}$ slices for immunofluorescence assays using a freezing microtome. The sections were incubated with anti-ionized calcium binding adapter molecule 1 (Iba1) (1 : 100; Abcam, Cambridge, UK) and anti-P2Y12 (1 : 50, Novus, Centennial, USA) as primary antibodies diluted in phosphate-buffered saline (PBS) overnight at $4^{\circ} \mathrm{C}$. After washing 3 times with PBS, the sections were incubated with Alexa 546-conjugated donkey anti-rabbit $(1: 200$; Thermo Fisher Scientific, Waltham, USA) and Alexa 488-conjugated donkey antigoat $(1: 200$, Thermo Fisher Scientific) antibodies for $2 \mathrm{~h}$. The sections were counterstained with 4,6-diamidino2-phenylindole (DAPI; Life Technologies, Grand Island, USA) to identify nuclei. Olympus LCX100 Imaging System (Olympus Corp., Tokyo, Japan) and ImageJ software (National Institutes of Health, Bethesda, USA) were used for image acquisition and analysis.

\section{Western blot analysis}

Heart tissue was homogenized in radioimmunoprecipitation assay (RIPA) lysis buffer containing 1\% phenylmethanesulfonyl fluoride (PMSF), and the supernatants were collected after centrifugation at $4^{\circ} \mathrm{C}$ and processed for western blot analysis. A BCA assay kit (Pierce Protein Biology, St. Louis, USA) was used to determine protein concentration. Approximately $70 \mu \mathrm{g}$ of total protein from each sample was resolved on $8-10 \%$ polyacrylamide gels and transferred to polyvinylidene fluoride (PVDF) membranes (BioRad, Richmond, USA). The membranes were incubated at $4^{\circ} \mathrm{C}$ overnight with anti-P2Y12 (1:2000; Abcam; ab184411), anti-platelet endothelial cell adhesion molecule-1 (CD31) (1 : 1000; Abcam; ab64543), anti-glyceraldehyde-3-phosphate dehydrogenase (anti-GAPDH) (1 : 5000; CoWin Bioscience, Beijing, China) antibodies diluted in general antibody dilution buffer. The blots were detected with an enhanced chemiluminescence (ECL) detection kit (Merck Millipore, Burlington, USA) and visualized using a FluorChem E Imager (Protein-Simple, Santa Clara, USA). The protein expression relative to GAPDH was analyzed using ImageJ software.

\section{Statistical analysis}

Data are presented as means \pm standard deviations (SD). Unpaired t-tests were used to compare values between 2 groups. Analysis of variance (ANOVA) and Tukey's test were used to compare the differences among multiple groups. The SPSS v. 17.0 software (SPSS Inc., Chicago, USA) was used for the analysis. A p-value less than 0.05 was considered significant in all statistical tests.

\section{Results}

\section{P2Y12 inhibition in macrophages attenuates infarct size following myocardial I/R injury in rats}

As shown in Fig. 1A,B, P2Y12 shRNA treatment significantly reduced ischemia size, as evidenced by Evans blue staining $(\mathrm{p}<0.05)$. The ECGs were examined to elucidate the physiological effect of I/R injury and the incidence rate of reperfusion arrhythmias. Successful ischemia injury was confirmed by ST-segment elevation, and reperfusion injury was confirmed by reduced ST-segment elevation and tall T-waves after ischemia. P2Y12 knockdown $(I / R+P)$ significantly reduced the incidence rates of reperfusion arrhythmias are shown in Fig. $1 \mathrm{C}$, and the duration of VT when compared with $\mathrm{I} / \mathrm{R}+\mathrm{V}$ as shown in Fig. 1D (23.07 \pm 4.32 compared to $55.83 \pm 11.3, \mathrm{p}<0.05)$. The incidence of reperfusion VT/VF was $90 \%$ in the I/R group; it was reduced to $50 \%$ following P2Y12 shRNA treatment.

\section{P2Y12 inhibition in macrophages attenuates inflammation induced by myocardial I/R injury in rats}

We used P2Y12 shRNA to determine the role of P2Y12R in macrophages following myocardial $I / R$ injury in rats. As shown in Fig. 2, immunohistochemical analysis of heart sections demonstrated a strong immunoreactive signal for Iba1 in the I/R areas in the control groups, which was significantly attenuated by P2Y12 shRNA treatment $(\mathrm{p}<0.05)$.

\section{P2Y12 inhibition in macrophages attenuates cardiac remodeling in rats}

To assess the role of P2Y12 in myocardial I/R injury, we employed the following strategies. To evaluate the efficacy of cardiac remodeling, we measured protein expression of $\alpha$-smooth muscle actin ( $\alpha$-SMA) and CD31 using immunohistochemistry and western blot analyses, respectively. We found that $\alpha$-SMA staining was significantly higher in the $I / R+P 2 Y 12$ shRNA group compared to the $I / R$ group (Fig. 3A). CD31 protein expression in the $I / R$ areas was significantly upregulated in the I/R + P2Y12 shRNA group compared to the other groups (Fig. 3B, 3C $0.31 \pm 0.01$ compared to $0.26 \pm 0.008$ (I/R + P compared to $\mathrm{I} / \mathrm{R}+\mathrm{V}$ ); $\mathrm{p}<0.05)$. The $\alpha$-SMA and CD31 staining also revealed increased vessel formation in the $I / R$ areas.

As shown in Fig. 3D, we observed that P2Y12 protein expression in the $I / R$ areas was significantly increased compared to the control groups $(0.23 \pm 0.01,0.17 \pm 0.007$ 
A

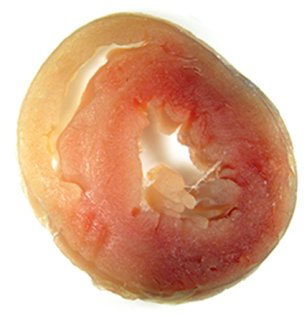

$\mathrm{C}+\mathrm{V}$

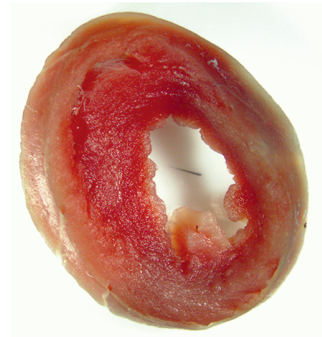

$\mathrm{C}+\mathrm{P}$

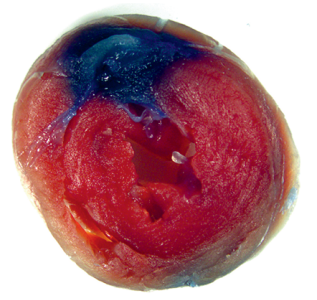

$\mathrm{I} / \mathrm{R}+\mathrm{V}$

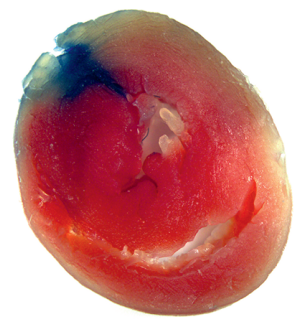

$\mathrm{I} / \mathrm{R}+\mathrm{P}$
B

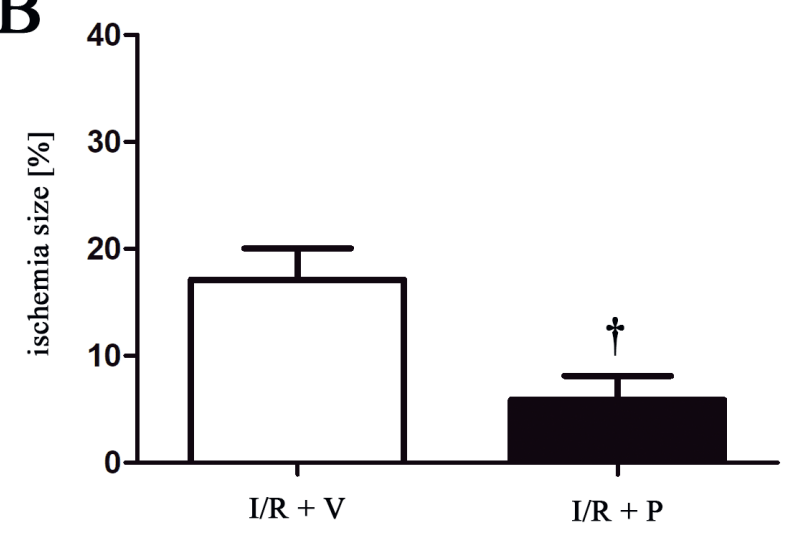

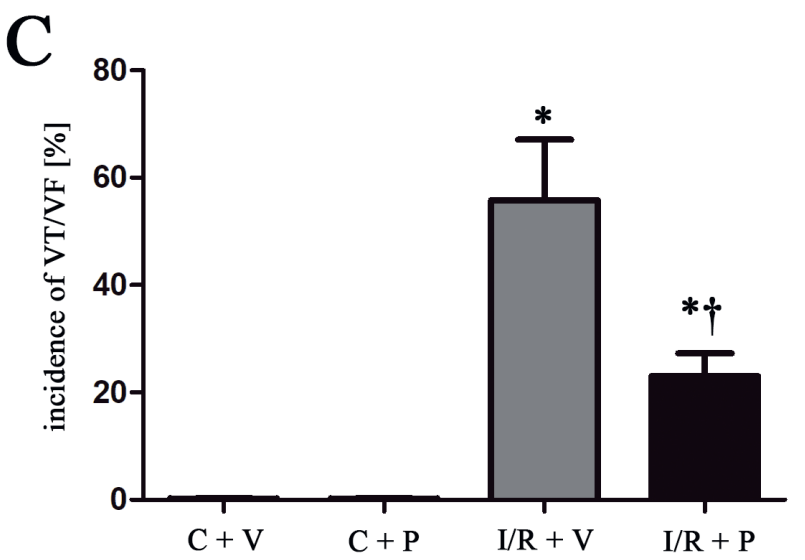

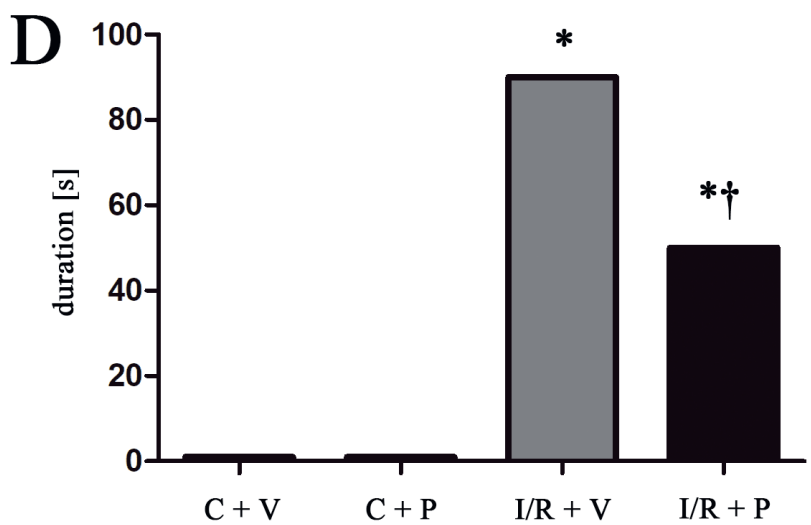

Fig. 1. A. Infarct and ischemic area as determined using Evans blue dye and TTC staining; B. Percent ischemic area after reperfusion; C. Comparisons of VT duration between the 4 groups after reperfusion; D. Comparisons of the incidence rates of reperfusion arrhythmias between the 4 groups after reperfusion; ${ }^{*} p<0.01$ compared with $C+V ;+p<0.05$ compared with I/R + V. C + V; control + vehicle; $C+P$, control + P2Y12 shRNA lentiviral vector; I/R + V, myocardial $\mathrm{I} / \mathrm{R}+$ vehicle; and I/R + P, myocardial I/R + P2Y12 shRNA lentiviral vector

compared to $0.150 \pm 0.007,0.16 \pm 0.009(\mathrm{I} / \mathrm{R}+\mathrm{V}$ and $\mathrm{I} / \mathrm{R}+\mathrm{P}$ compared to $\mathrm{C}+\mathrm{V}$ and $\mathrm{C}+\mathrm{P}) ; \mathrm{p}<0.05)$. The $\mathrm{I} / \mathrm{R}+\mathrm{P} 2 \mathrm{Y} 12$ shRNA group had significantly lower P2Y12 protein expression compared to the I/R group $(0.17 \pm 0.007$ compared to $0.23 \pm 0.01(\mathrm{I} / \mathrm{R}+\mathrm{P}$ compared to $\mathrm{I} / \mathrm{R}+\mathrm{V})$; $\mathrm{p}<0.05)$.

\section{Discussion}

The present study was designed to investigate the role of P2Y12 in macrophages following myocardial I/R injury in rats. The primary findings of our study are as follows: 1) P2Y12 shRNA lentiviral vector reduced P2Y12 protein expression in macrophages; 2) reperfusion treatment significantly increased the incidence of arrhythmias, and inhibiting P2Y12 in macrophages improved the duration and incidence of arrhythmias; and 3) inhibiting P2Y12 reduced inflammation associated with reperfusion.

Myocardial ischemia can lead to cardiovascular disease and AMI, which is the major cause of death worldwide. Reperfusion is the primary treatment for AMI, and timely and successful reperfusion can reduce myocardial ischemia injury, limit infarct areas and improve ventricular function. However, tissue ischemia often occurs despite the fact that the flow of blood is restored, resulting in myocardial I/R injury, which is characterized by endothelial cell 
A

DAPI
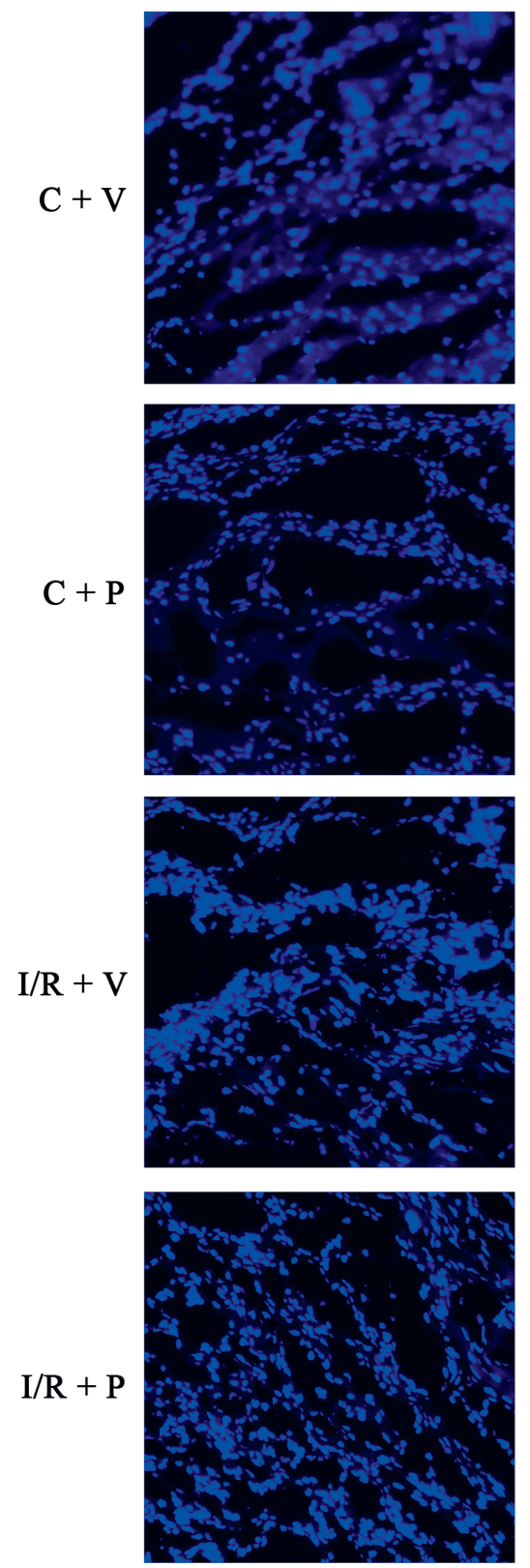

B

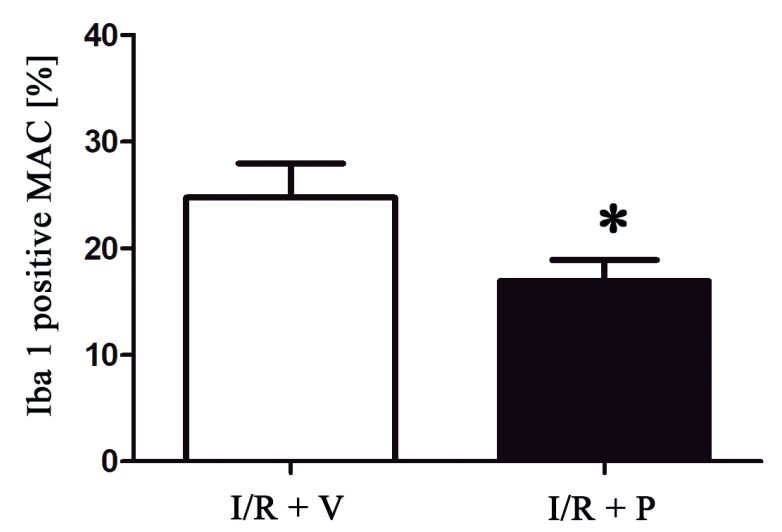

Iba 1
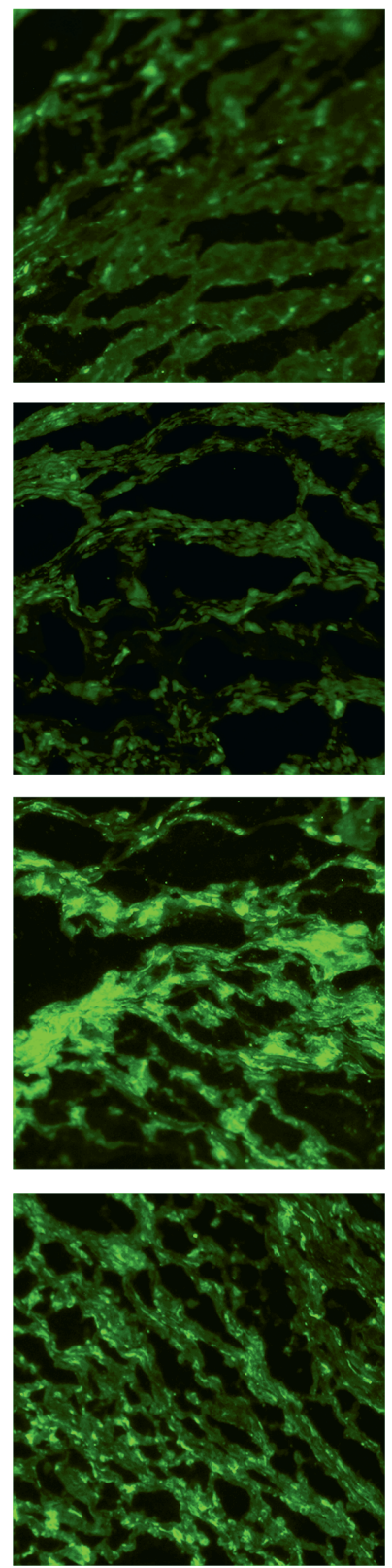

P2Y12
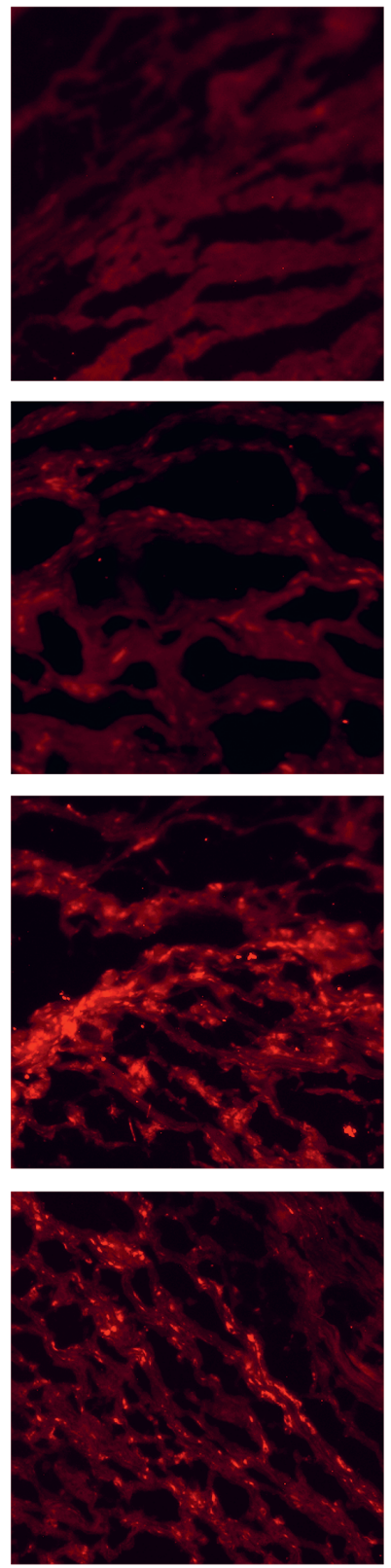
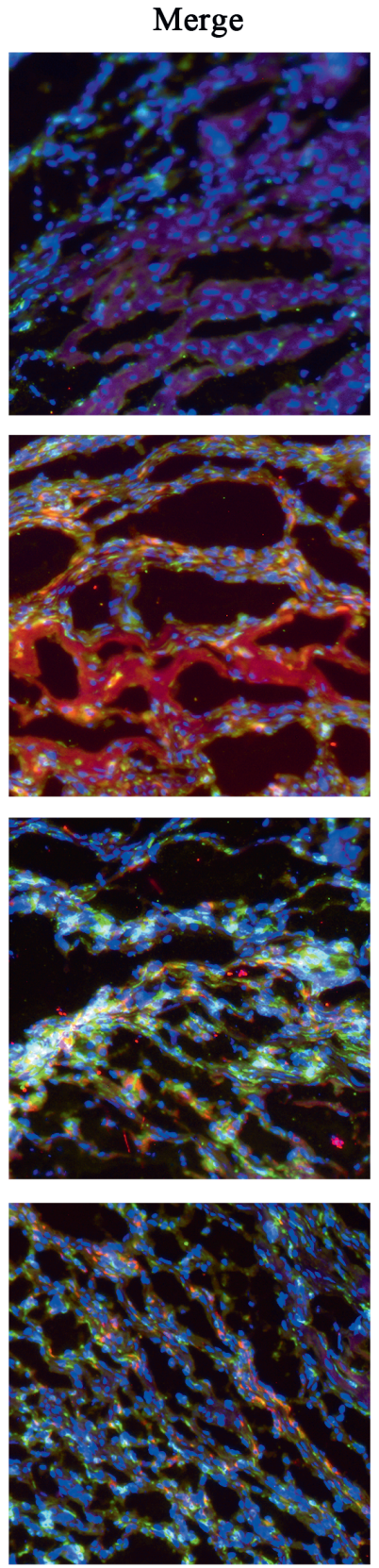

C

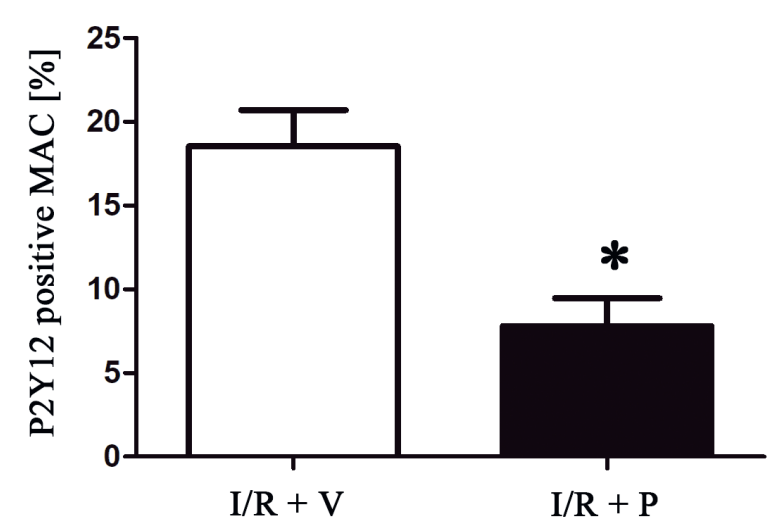

Fig. 2. A. Representative double-immunostained images of the macrophage marker lba1 (green) and P2Y12 (red); last column shows merged images of all staining in the 4 groups after reperfusion; B. Percent of Iba1 expressed in the ischemic areas; C. Percent of P2Y12 expressed in the ischemic area. Values shown are the mean $\pm S D ;{ }^{*} p<0.01$ compared with I/R $+\mathrm{V} ; \mathrm{C}+\mathrm{V}$, control + vehicle; $\mathrm{C}+\mathrm{P}$, control $+\mathrm{P} 2 \mathrm{Y} 12$ shRNA lentiviral vector; I/R $+\mathrm{V}$, myocardial I/R + vehicle; and I/R + P, myocardial I/R + P2Y12 shRNA lentiviral vector 


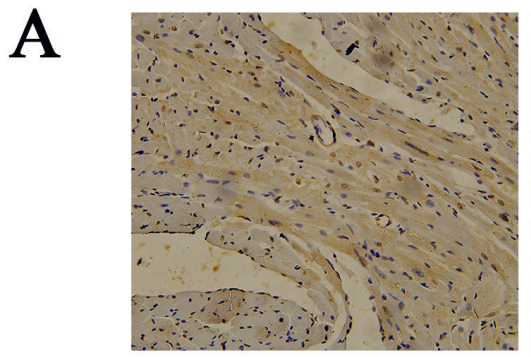

$\mathrm{C}+\mathrm{V}$

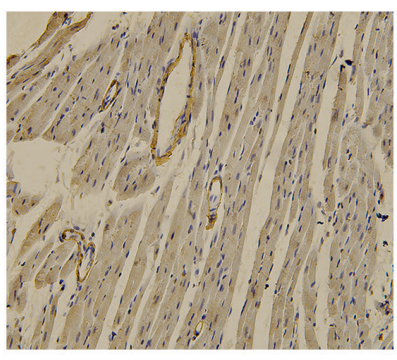

$\mathrm{C}+\mathrm{P}$

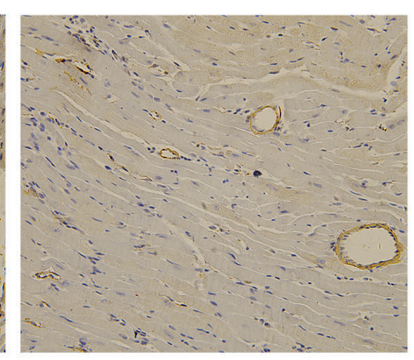

$\mathrm{I} / \mathrm{R}+\mathrm{V}$

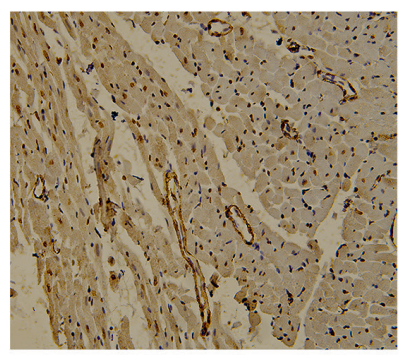

$\mathrm{I} / \mathrm{R}+\mathrm{P}$

B

CD31

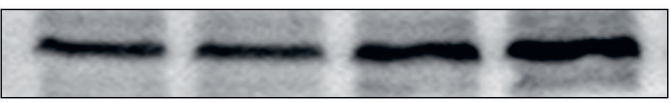

GAPDH

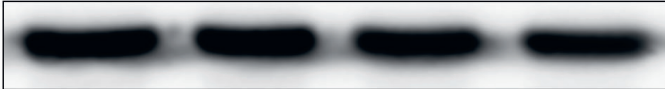

C

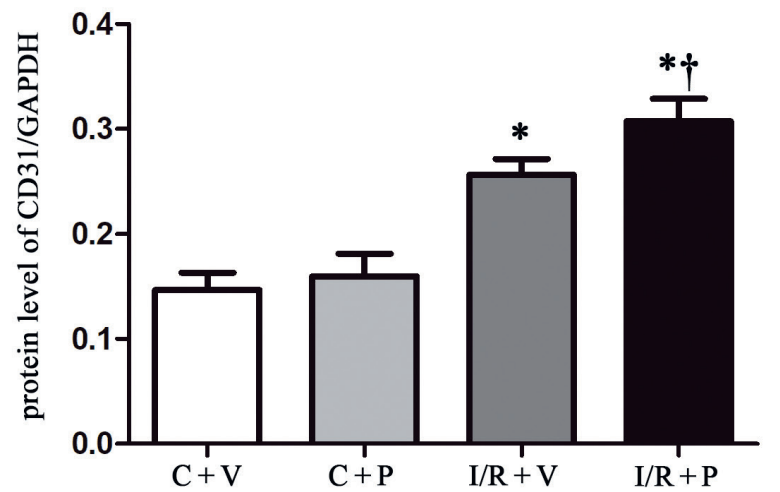

$\mathrm{D}$

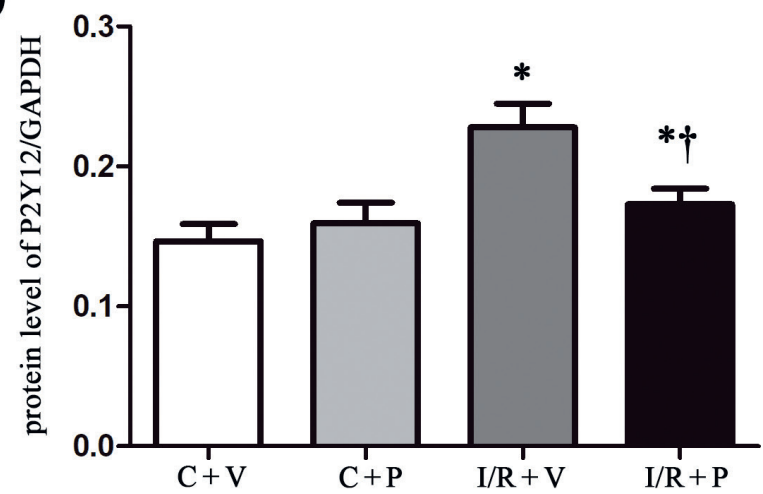

Fig. 3. A. Immunohistochemical staining of a-SMA in the 4 groups after reperfusion; B. Representative western blot images of CD31, P2Y12 and GAPDH expression in the 4 groups after reperfusion; C. Quantified CD31 expression relative to GAPDH expression in the 4 groups; D. Quantified P2Y12 expression relative to GAPDH expression in the 4 groups; ${ }^{*} p<0.01$ compared with $C+V_{i}+p<0.05$ compared with $I / R+V$

dysfunction, DNA damage and inflammation..$^{9}$ A variety of clinical conditions can cause I/R injury, such as myocardial injury, stroke, organ transplantation, limb ischemia, and multiple organ system dysfunction. In the last 20 years, it has been confirmed that inflammation plays a vital role in the pathophysiological process of I/R injury. Inflammation may induce ectopic triggers, leading to the occurrence of arrhythmias. ${ }^{9} 12$ Recent studies provide evidence that immune cells are involved in I/R-induced wound healing. ${ }^{13-18}$ Macrophages are the primary inflammatory cell responders, and they play an important role in myocardial healing following infarction, ${ }^{19}$ as well as the inflammatory response after I/R injury. ${ }^{20}$

The P2Y12 receptor is composed of 2 parts: seventransmembrane (7-TM) $\alpha$-helix bundle and a carboxyl terminal helix. ${ }^{20} \mathrm{P} 2 \mathrm{Y} 12$ is expressed in platelets, where it stabilizes platelet aggregation induced by thrombin and thromboxane A2 (TXA2) and other agonists, and also plays an important role in thrombosis in vivo. ${ }^{21-24}$ Activation of the ADP-P2Y12 pathway can induce an inflammatory state in vascular smooth muscle and lead to atherosclerosis. ${ }^{25} \mathrm{~A}$ series of studies have shown that P2Y12 antagonists have cardioprotective properties that are independent of their anti-thrombotic actions. ${ }^{26}$ This protective effect is mainly due to adenosine receptor activation and downstream phosphorylation of protein kinase $B$ and endothelial nitric oxide synthase, and activation of cyclooxygenase- $2 .{ }^{27}$ Adenosine plays a key protective role during myocardial I/R injury. ${ }^{28-31}$ Blocking the P2Y12 receptor can increase extracellular adenosine levels by inhibiting the reuptake of interstitial adenosine in cells. ${ }^{32,33}$ The present study intended to investigate the cardioprotective effects of P2Y12 in the context of inflammation.

P2Y12 is also expressed on macrophages. A P2Y12 antagonist has been shown to attenuate atherogenesis and reduce accumulation of macrophages in ApoE-deficient mice. ${ }^{34}$ A recent study demonstrated that P2Y12 expression in macrophages was involved in the modulation of the immune microenvironment in various pathological inflammatory conditions, a fact that indicates that P2Y12 is an important immunomodulatory receptor on macrophages. ${ }^{35}$ In the present study, shRNA was used to knock down 
P2Y12 expression in macrophages. Systemic administration of P2Y12 shRNA had no effect on platelet expression, given that platelets lack nuclei. Thus, we were able to rule out the effect of blocking P2Y12 in platelets in our I/R model. Our immunofluorescence staining of P2Y12 in macrophages demonstrated that P2Y12 plays an anti-inflammatory role in I/R injury. Activation of P2Y12 in macrophages could induce cell proliferation and lamellar foot formation, and inhibiting P2Y12 could reduce chemotaxis. ${ }^{36}$ Our results suggest that regulating P2Y12 in macrophages could improve the duration and incidence rates of reperfusion arrhythmias through 2 possible mechanisms.

Downregulating P2Y12 in macrophages could ameliorate the immune microenvironment and exert anti-inflammatory effects in the I/R injury area. Furthermore, since cardiac tissue inflammation is related to cardiovascular sympathetic tone, sympathetic overstimulation and inflammation could be 2 major mediators of reperfusion arrhythmias. ${ }^{5}$

Another possibility is that reperfusion arrhythmias were obviously correlated with myocardial I/R injury. Our results showed that systemic administration of P2Y12 shRNA could significantly reduce the ischemia area, altering the release of oxygen free radicals and inflammatory factors.

In our study, we only carried out animal experiments to investigate the cardioprotective effect of P2Y12 on macrophages. However, determination of the effect of P2Y12 inhibition on macrophages in clinical scenarios and the exact mechanism underlying the effect requires further research. Ticagrelor is a P2Y12 receptor antagonist with proven clinical benefits in patients with myocardial infarction and acute coronary syndrome. ${ }^{37}$ This experiment elaborated upon the mechanism of potential cardioprotective effects outside of the antiplatelet actions of ticagrelor, particularly from the perspective of inflammation regulation.

\section{Limitations}

In our study, we only carried out animal experiments to investigate the cardioprotective effect of P2Y12 on macrophages. However, determination of the effect of P2Y12 inhibition on macrophages in clinical scenarios and the exact mechanism underlying the effect requires further research. Ticagrelor is a P2Y12 receptor antagonist with proven clinical benefits in patients with myocardial infarction and acute coronary syndrome. ${ }^{37}$ This experiment elaborated upon the mechanism of potential cardioprotective effects outside of the antiplatelet actions of ticagrelor, particularly from the perspective of inflammation regulation.

\section{Conclusions}

Knockdown of P2Y12 in macrophages was a main mechanism in improving the duration and incidence of arrhythmias after reperfusion in a I/R rat model. In addition, blocking P2Y12 could decrease the inflammatory response after cardiac reperfusion, which may be a new mechanism to target in the development of new cardioprotective therapeutics.

\section{ORCID iDs}

Lu Wang (1) https://orcid.org/0000-0003-2076-0624

$\mathrm{Na}$ Li (1) https://orcid.org/0000-0001-7087-6495

Fei Wang (10) https://orcid.org/0000-0001-5817-2161

Lianqun Cui (1) https://orcid.org/0000-0003-2888-0639

\section{References}

1. Park ES, Kang DH, Kang JC, et al. Cardioprotective effect of KR-33889, a novel PARP inhibitor, against oxidative stress-induced apoptosis in H9c2 cells and isolated rat hearts. Arch Pharm Res. 2017;40(5): 640-654. doi:10.1007/s12272-017-0912-3

2. Di Lisa F, Bernardi P. Mitochondria and ischemia-reperfusion injury of the heart: Fixing a hole. Cardiovasc Res. 2006;70(2):191-199. doi:10. 1016/j.cardiores.2006.01.016

3. Chen YF, Chen WY, Chung CH, Kuo CL, Lee AS. Cardiac protection of Bauhinia championii against reperfusion injury. Environ Toxicol. 2020;35(7):774-782. doi:10.1002/tox.22912

4. Mehta RH, Starr AZ, Lopes RD, et al. Relationship of sustained ventricular tachyarrhythmias to outcomes in patients undergoing primary percutaneous coronary intervention with varying underlying baseline risk. Am Heart J. 2011;161(4):782-789. doi:10.1016/j.ahj.2011.01.005

5. Wang S, Wu L, Li X, et al. Light-emitting diode therapy protects against ventricular arrhythmias by neuro-immune modulation in myocardial ischemia and reperfusion rat model. J Neuroinflammation. 2019;16(1): 139. doi:10.1186/s12974-019-1513-5

6. Troidl C, Mollmann $\mathrm{H}, \mathrm{Nef} \mathrm{H}$, et al. Classically and alternatively activated macrophages contribute to tissue remodelling after myocardial infarction. J Cell Mol Med. 2009;13(9B):3485-3496. doi:10.1111/ j.1582-4934.2009.00707.x

7. Outtz HH, Wu JK, Wang X, Kitajewski J. Notch1 deficiency results in decreased inflammation during wound healing and regulates vascular endothelial growth factor receptor-1 and inflammatory cytokine expression in macrophages. J Immunol. 2010;185(7):4363-4373. doi:10.4049/jimmunol.1000720

8. Zhang K, Zhang J, Gao ZG, et al. Structure of the human P2Y12 receptor in complex with an antithrombotic drug. Nature. 2014;509(7498): 115-118. doi:10.1038/nature13083

9. Ye Y, Birnbaum GD, Perez-Polo JR, Nanhwan MK, Nylander S, Birnbaum Y. Ticagrelor protects the heart against reperfusion injury and improves remodeling after myocardial infarction. Arterioscler Thromb Vasc Biol. 2015;35(8):1805-1814. doi:10.1161/ATVBAHA.115.305655

10. Rao DD, Vorhies JS, Senzer N, Nemunaitis J. siRNA vs. shRNA: Similarities and differences. Adv Drug Deliv Rev. 2009;61(9):746-759. doi:10. 1016/j.addr.2009.04.004

11. Liverani E, Rico MC, Garcia AE, Kilpatrick LE, Kunapuli SP. Prasugrel metabolites inhibit neutrophil functions. J Pharmacol Exp Ther. 2013;344(1):231-243. doi:10.1124/jpet.112.195883

12. Nicoletti A, Michel JB. Cardiac fibrosis and inflammation: Interaction with hemodynamic and hormonal factors. Cardiovasc Res. 1999; 41(3):532-543. doi:10.1016/s0008-6363(98)00305-8

13. Dong X, Swaminathan S, Bachman LA, Croatt AJ, Nath KA, Griffin MD. Resident dendritic cells are the predominant TNF-secreting cell in early renal ischemia-reperfusion injury. Kidney Int. 2007;71(7):619-628. doi:10.1038/sj.ki.5002132

14. Walsh SK, Kane KA, Wainwright CL. Mast cells, peptides and cardioprotection: An unlikely marriage? Auton Autacoid Pharmacol. 2009; 29(3):73-84. doi:10.1111/j.1474-8673.2009.00436.x

15. Meldrum DR. Tumor necrosis factor in the heart. Am J Physiol. 1998; 274(3):R577-R595. doi:10.1152/ajpregu.1998.274.3.R577

16. Levick SP, Gardner JD, Holland M, Hauer-Jensen M, Janicki JS, Brower GL. Protection from adverse myocardial remodeling secondary to chronic volume overload in mast cell deficient rats. J Mol Cell Cardiol. 2008; 45(1):56-61. doi:10.1016/j.yjmcc.2008.04.010

17. Linfert $\mathrm{D}$, Chowdhry $\mathrm{T}$, Rabb H. Lymphocytes and ischemia-reperfusion injury. Transplant Rev (Orlando). 2009;23(1):1-10. doi:10.1016/j. trre.2008.08.003 
18. Lahat N, Rahat MA, Ballan M, Weiss-Cerem L, Engelmayer M, Bitterman $\mathrm{H}$. Hypoxia reduces CD80 expression on monocytes but enhances their LPS-stimulated TNF-alpha secretion. J Leukoc Biol. 2003;74(2):197-205. doi:10.1189/jlb.0303105

19. Frantz S, Hofmann U, Fraccarollo D, et al. Monocytes/macrophages prevent healing defects and left ventricular thrombus formation after myocardial infarction. FASEB J. 2013;27(3):871-881. doi:10.1096/ fj.12-214049

20. Pruenster M, VoglT, Roth J, Sperandio M. S100A8/A9: From basic science to clinical application. Pharmacol Ther. 2016;167:120-131. doi:10. 1016/j.pharmthera.2016.07.015

21. Cattaneo M. P2Y12 receptors: Structure and function. JThromb Haemost. 2015;13(Suppl 1):S10-S16. doi:10.1111/jth.12952

22. Crepaldi P, Cacciari B, Bonache MC, et al. 6-amino-2-mercapto-3H-pyrimidin-4-one derivatives as new candidates for the antagonism at the P2Y12 receptors. Bioorg Med Chem. 2009;17(13):4612-4621. doi:10.1016/j.bmc.2009.04.061

23. Cattaneo M, Canciani MT, Lecchi A, et al. Released adenosine diphosphate stabilizes thrombin-induced human platelet aggregates. Blood. 1990;75(5):1081-1086. PMID:2137716

24. Eckly A, Gendrault JL, Hechler B, Cazenave JP, Gachet C. Differential involvement of the P2Y1 and P2YT receptors in the morphological changes of platelet aggregation. Thromb Haemost. 2001;85(4):694-701. PMID:11341507

25. Rauch BH, Filep JG. Purinergic receptors and atherosclerosis: Emerging role for vessel wall P2Y12. Cardiovasc Res. 2014;102(3):339-341. doi:10.1093/cvr/cvu108

26. Yang XM, Liu Y, Cui L, et al. Platelet P2Y(1)(2) blockers confer direct postconditioning-like protection in reperfused rabbit hearts. J CardiovasC Pharmacol Ther. 2013;18(3):251-262. doi:10.1177/1074248412467692

27. Nanhwan MK, Ling S, Kodakandla M, Nylander S, Ye Y, Birnbaum Y. Chronic treatment with ticagrelor limits myocardial infarct size: An adenosine and cyclooxygenase-2-dependent effect. Arterioscler Thromb Vasc Biol. 2014;34(9):2078-2085. doi:10.1161/ATVBAHA.114.304002
28. Armstrong D, Summers C, Ewart L, Nylander S, Sidaway JE, van Giezen JJ. Characterization of the adenosine pharmacology of ticagrelor reveals therapeutically relevant inhibition of equilibrative nucleoside transporter 1. J Cardiovasc Pharmacol Ther. 2014;19(2):209-219. doi:10.1177/ 1074248413511693

29. Bonello L, Laine M, Kipson N, et al. Ticagrelor increases adenosine plasma concentration in patients with an acute coronary syndrome. J Am Coll Cardiol. 2014;63(9):872-877. doi:10.1016/j.jacc.2013.09.067

30. van Giezen JJ, Sidaway J, Glaves P, Kirk I, Bjorkman JA. Ticagrelor inhibits adenosine uptake in vitro and enhances adenosine-mediated hyperemia responses in a canine model. J Cardiovasc Pharmacol Ther. 2012;17(2):164-172. doi:10.1177/1074248411410883

31. Cattaneo M, Schulz R, Nylander S. Adenosine-mediated effects of ticagrelor: Evidence and potential clinical relevance. J Am Coll Cardiol. 2014;63(23):2503-2509. doi:10.1016/j.jacc.2014.03.031

32. Kitakaze M, Minamino T, Node K, et al. Adenosine and cardioprotection in the diseased heart. Jpn Circ J. 1999;63(4):231-243. doi:10.1253/ jcj.63.231

33. Kloner RA. Current state of clinical translation of cardioprotective agents for acute myocardial infarction. Circ Res. 2013;113(4):451-463. doi:10.1161/CIRCRESAHA.112.300627

34. Ganbaatar B, Fukuda D, Salim HM, et al. Ticagrelor, a P2Y12 antagonist, attenuates vascular dysfunction and inhibits atherogenesis in apolipoprotein-E-deficient mice. Atherosclerosis. 2018;275:124-132. doi:10.1016/j.atherosclerosis.2018.05.053

35. Kloss L, Dollt C, Schledzewski K, et al. ADP secreted by dying melanoma cells mediates chemotaxis and chemokine secretion of macrophages via the purinergic receptor P2Y12. Cell Death Dis. 2019;10(10): 760. doi:10.1038/s41419-019-2010-6

36. Kronlage $M$, Song J, Sorokin $L$, et al. Autocrine purinergic receptor signaling is essential for macrophage chemotaxis. Sci Signal. 2010; 3(132):ra55. doi:10.1126/scisignal.2000588

37. Moulias A, Xanthopoulou I, Alexopoulos D. Does ticagrelor improve endothelial function? J Cardiovasc Pharmacol Ther. 2019;24(1):11-17. doi:10.1177/1074248418786936 